\title{
Le levé topographique haute densité par scanner laser 3D appliqué au génie côtier : l'exemple du suivi des digues du port de Bayonne
}

\author{
Bertrand CHAZALY ${ }^{1}$, David LHOMME ${ }^{1}$, Yvan ROBIN ${ }^{1}$ \\ Simon FAGES ${ }^{2}$, Stéphane GUBERT ${ }^{2}$ \\ ${ }^{1}$ ATM3D sarl \\ 16 av. du Midi, ZAC de Tourel, 30111 Congénies, France \\ contact@atm3d.com \\ ${ }^{2}$ Conseil Régional d'Aquitaine, Service du Développement et de l'Exploitation \\ du Port de Bayonne, Unité Etudes et Travaux Maritimes \\ 8 avenue de l'Adour, 64600 ANGLET, France
}

\section{Résumé :}

Le renforcement des talus d'enrochements des digues du port de Bayonne nécessitait une connaissance précise des volumes et des zones à recharger. Mais la géométrie accidentée de ces talus et la longueur des digues rendaient très fastidieuse toute tentative de levé topographique par méthode classique. Le scanner laser 3D est une nouvelle génération de capteur topographique permettant d'enregistrer plusieurs millions de points en $3 \mathrm{D}$, en quelques minutes, à des portées dépassant plusieurs centaines de mètres, à une précision centimétrique. Le levé lasergrammétrique des digues en 2004 et 2007, couplé au levé bathymétrique de l'embouchure, a fourni les images 3D d'où ont été extraites les informations indispensables à l'étude du comportement des digues.

\begin{abstract}
:
Traditional land surveying was not suitable to the Bayonne sea walls (South of France), because of their length and shape. In order to get quickly accurate information about volumes and displacement, 3D laser scanning was used, in 2004 and 2007. Linked to bathymetric survey, the very high density of the captured information has produced complete and accurate 3D images of the walls, used to point out changing areas and calculate volumes of repair.
\end{abstract}

Mots-clés :

Génie côtier - Travaux maritimes - Digue - Enrochement - Topographie Scanner Laser 3D - Lasergrammétrie - Bathymétrie - Modélisation numérique 


\section{$1 \quad$ Introduction}

La grande digue Nord de Tarnos, construite en 1966 et renforcée une première fois en 1987 constitue le principal ouvrage de protection de l'accès au port de Bayonne, auquel s'ajoutent la digue Intérieure et la digue Sud, au sud de l'embouchure. Ces trois digues représentent un linéaire cumulé de près de $1500 \mathrm{~m}$, soit $3000 \mathrm{~m}$ de talus.

Attaqués par l'action des houles, les talus d'enrochements naturels ou de blocs artificiels en béton ont évolué, se sont raidis et présentent des creux et donc une stabilité diminuée. Pour mettre au point le projet de renforcement il est nécessaire de connaître les quantités de matériaux à prévoir pour ramener l'ouvrage à sa géométrie initiale.

Il était donc nécessaire de disposer de la géométrie précise des ouvrages de façon à évaluer finement les volumes d'enrochements manquants sur la carapace afin de quantifier précisément son rechargement et de pouvoir analyser les mouvements des blocs artificiels en béton ainsi que les enrochements ophitiques.

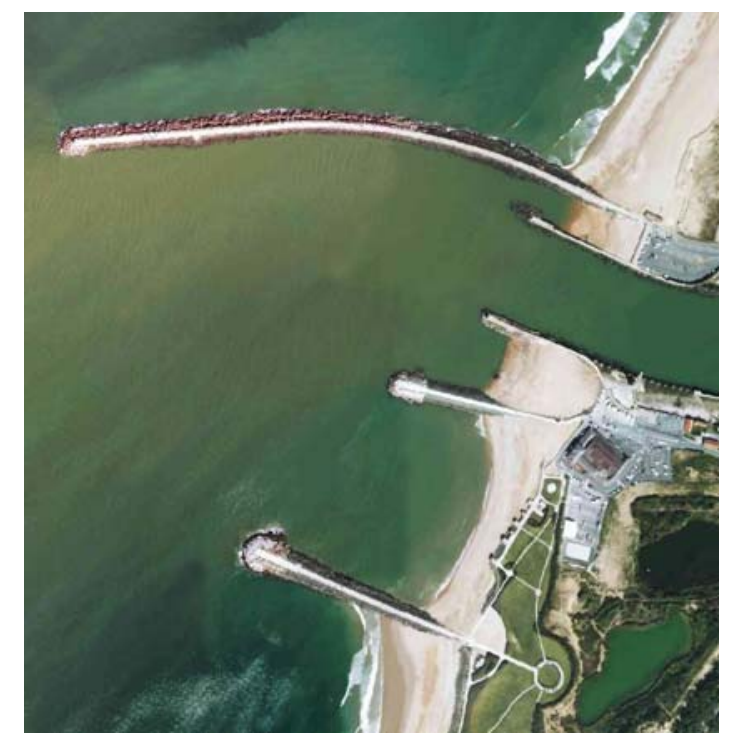

Figure 1. Les 3 digues protégeant l'accès au port de Bayonne

\section{Levé topographique : quelle solution?}

\subsection{Objectifs}

Le levé topographique avait pour but de dresser un état des lieux, un état référent de la digue nord de façon à le comparer aux mesures antérieures (données ponctuelles) et d'engager pour l'avenir un suivi global et formalisé de l'ouvrage. Cette campagne topographique devait servir également à lever des profils de la digue permettant d'évaluer, en les superposant aux profils théoriques de l'ouvrage, 
les pertes de matériaux rencontrées (pentes et volumes des talus) et les désordres qu'ils engendrent. La finalité était d'identifier les pathologies de l'ouvrage, métrer et chiffrer le renforcement des talus.

Les profils devaient être implantés en des points précis correspondant, entre autre, aux endroits déjà recensés lors d'une visite d'inspection en juin 2002 comme étant des sites particulièrement soumis à la houle et au courant, c'est à dire des lieux ayant certainement subis des désordres significatifs dans le temps.

Ces profils devaient permettre de visualiser les mouvements de l'ouvrage et servir de référentiel pour la mise en place d'un suivi ultérieur.

\subsection{Problématique}

Pour évaluer finement les matériaux déficitaires sur les talus et positionner précisément les zones de rechargement lors du confortement des talus, il fallait mettre en œuvre une méthode de mesure qui permette d'obtenir rapidement une densité de points suffisante.

Le levé topographique traditionnel s'adaptait difficilement aux profils accidentés de l'ouvrage et aux conditions marines :

- La distance entre les digues et le manque de vis-à-vis empêchent la mesure au distancemètre sans prisme.

- L'accès des opérateurs avec un prisme réflecteur nécessite le recours à des équipes spécialisées pour se déplacer dans des talus de blocs cubiques de plus de $2.50 \mathrm{~m}$ d'arête (BCR, Bloc Cubique Rainuré de 40 tonnes).

- Pour espérer acquérir un maximum d'information sur les parties émergées des digues, la mesure doit être réalisée à marée basse, pendant les grandes marées d'automne, ce qui réduit le temps d'acquisition à quelques jours.

Un levé par méthode photogrammétrique aurait permis un enregistrement rapide, mais la richesse de l'information à extraire et la géométrie complexe des digues auraient rendu ce travail très laborieux.

Les méthodes classiques auraient donc conduit à des coûts prohibitifs interdisant un relevé exhaustif.

La récente technologie de mesure par scanner 3D semblait répondre au mieux au besoin de densité et de précision, en assurant la rapidité d'acquisition indispensable à un enregistrement complet en quelques jours.

\subsection{La solution du scanner laser 3D}

Le levé à l'aide d'un laser 3D est apparu comme étant la solution la plus pertinente au vu de ces performances, rapidité d'exécution, précision, rendu de l'information 
et de son traitement, pour un coût raisonnable. Encore peu mise en cuvre en France, cette technologie tend à être de plus en plus utilisée pour l'auscultation des ouvrages en enrochement (CIRIA et al. 2007). D'autre part, cette technique est d'autant plus intéressante qu'elle se marie parfaitement avec des sondages bathymétriques multi-faisceaux. En effet, le format des données produites par un scanner 3D est similaire à celui d'une campagne de levé bathymétrique. Si les deux acquisitions sont réalisées dans le même système de coordonnées, leur fusion est automatique et permet une vue très précise de l'ouvrage dans sa globalité et dans son environnement.

\section{Le levé lasergrammétrique}

\subsection{La technologie}

La lasergrammétrie, ou levé scanner laser 3D, est une nouvelle technologie qui bouleverse les méthodes de relevé. Elle utilise une nouvelle génération d'instruments de mesure, les scanners 3D, capables d'enregistrer plusieurs millions de points en trois dimensions en quelques minutes, avec une précision de quelques millimètres et une portée dépassant pour certains plusieurs centaines de mètres. Le bond technologique réside dans la capacité de ce type d'appareil à collecter un très grand nombre d'informations très rapidement et à distance.

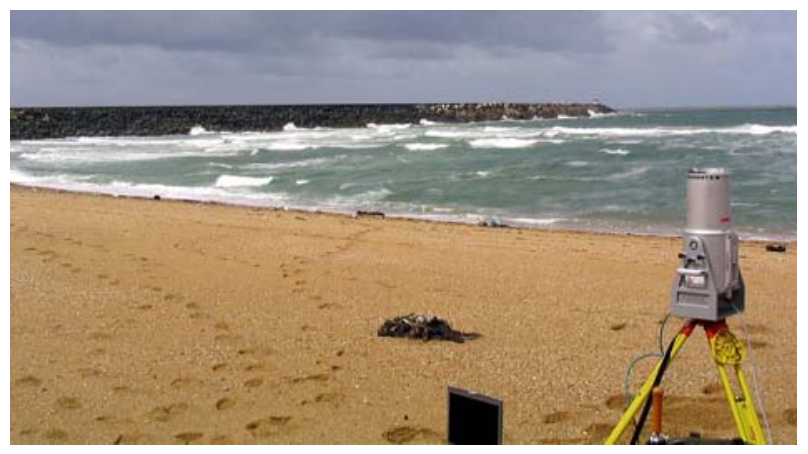

Fig. 2 : Le scanner Riegl LMS 420i numérisant la digue nord

Le résultat d'une opération de numérisation est avant tout un fichier numérique contenant une série de coordonnées géométriques $(\mathrm{X}, \mathrm{Y}, \mathrm{Z})$, constituant ce qu'on appelle un « nuage de points » 3D. Selon les besoins exprimés, le traitement des nuages de points sera différent. Il nécessite toujours l'utilisation de logiciels spécifiques, développés exclusivement pour la gestion de données 3D denses (CHAZALY, 2007). 


\subsection{Mise en ouvre à Bayonne}

L'entreprise ATM3D est intervenue à deux reprises à Bayonne : la première fois en 2004 sur la digue nord et la digue intérieure, la seconde fois en novembre 2007 pour numériser les trois digues. Chaque intervention a duré 6 jours, au cours desquels l'intégralité de l'enveloppe extérieure des digues a été numérisée en 3D. Le scanner utilisé à Bayonne était le Riegl LMS Z 420i. Enregistrant à une vitesse de 10.000 points par seconde, sa portée de plus de $700 \mathrm{~m}$ a permis d'acquérir des points à travers l'embouchure de l'Adour, par exemple depuis la digue Nord vers la digue Sud et inversement. La numérisation a été réalisée pendant les phases de plus basse marée (jour et nuit) par une équipe de trois techniciens : un ingénieur, un opérateur scanner 3D et un géomètre topographe.

Quarante et une stations ont été réalisées. Pour chacune, une acquisition panoramique faible densité a été enregistrée ( 280.000 points par position) puis une fenêtre beaucoup plus dense a été faite sur les ouvrages, à raison d'un point tous les $5 \mathrm{~cm}$ en moyenne (plusieurs millions de points par fenêtre).

Pour les musoirs et l'extrémité Ouest des digues Nord et Sud, le manque de positions en vis à vis a entraîné des masques sur l'enrochement et les blocs, de sorte que les mesures ont rarement couvert les faces des blocs orientées vers l'océan. Cependant, les faces orientées côté digue sont couvertes par suffisamment de points pour caractériser les mouvements des blocs.

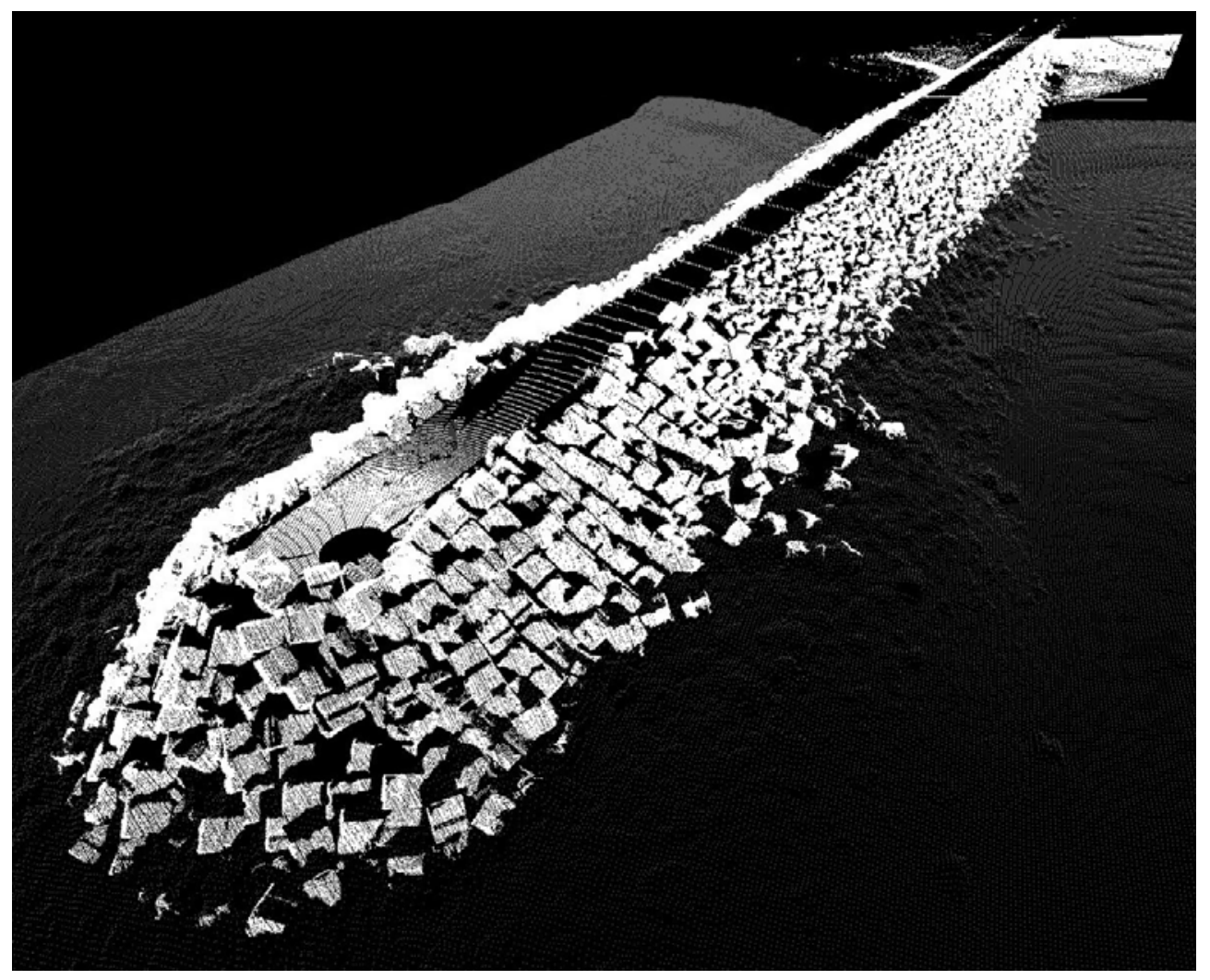


Figure 3. Extrait d'un nuage de points 3D acquis à Bayonne

Avant filtrage et échantillonnage, ce sont 100 millions de points 3D qui ont été enregistrés à la surface des 3 digues.

Les mesures terrestres ont été assemblées en trois nuages de points (un nuage par digue). Chaque nuage a été filtré automatiquement afin d'éliminer les points parasites dus aux embruns et à la présence des promeneurs et des pêcheurs dans le champ du scanner. Les 3 nuages de points ont ensuite été numériquement assemblés aux mesures bathymétriques, sans affinage du calage. La précision des calages topographiques suffit pour une bonne superposition des deux types de mesures. On constate qu'elles se recouvrent sur une hauteur de 1 mètre environ.

Le tout a été coloré en fonction de la hauteur et mis en forme pour une visualisation 3D des résultats sous une visionneuse fournie avec les données et permettant :

- la visualisation et le partage des mesures laser 3D (nuages de points denses),

- la visualisation et le partage de modèles polygonaux,

- la navigation à travers les résultats de numérisation 3D (avancer, zoomer, etc.),

- la prise des mesures interactives telles que des angles, des distances, des rayons, des coordonnées,

- le positionnement de plans de coupe et l'extraction à volonté de profils DXF,

- la génération de rapports d'inspection au format Word, Excel ou HTML.

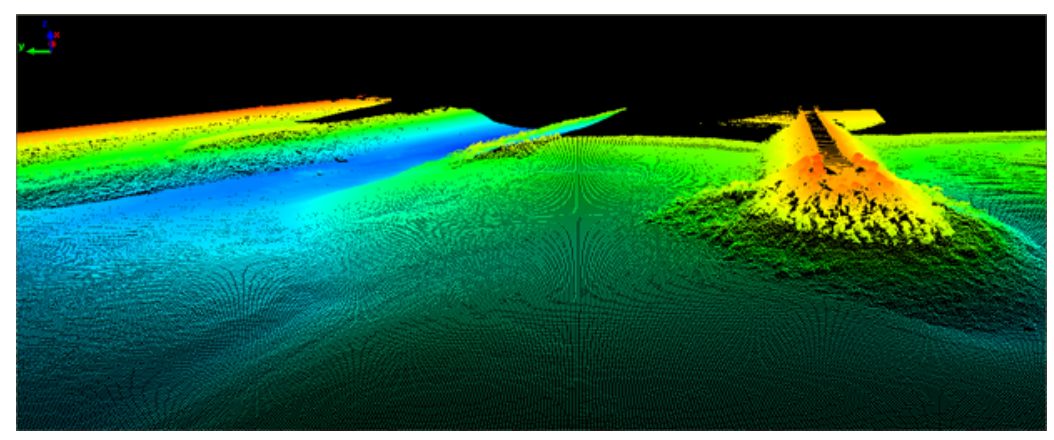

Figure 4. Superposition levé terrestre haute densité et levé bathymétrique

Cent cinquante profils en travers ont été extraits des nuages, cartographiés et mis en forme sous forme de cahiers de profils Autocad. Superposés aux profils théoriques des digues, ils ont permis de calculer le volume précis de l'enrochement manquant. 
Enfin, les données 2007 et les données 2004 ont été superposées et fournies avec la visionneuse 3D, pour visualiser en 3D le déplacement des blocs.

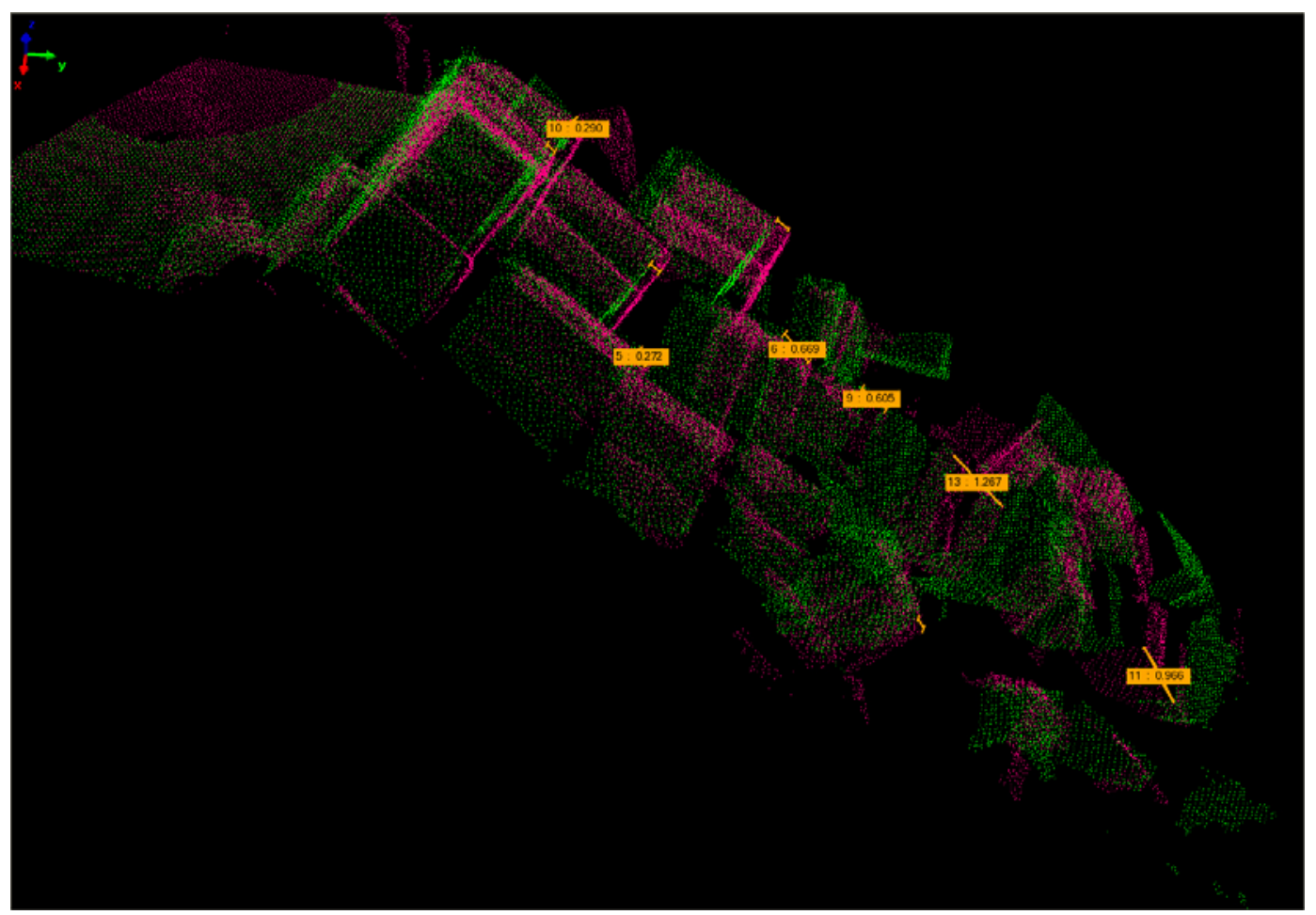

Figure 5. Superposition des mesures 2004(en vert) et 2007(en fushia)

\section{Conclusions}

$\mathrm{Au}$ vu des différences ponctuelles d'altitude, en raison de la taille des blocs rencontrés (BCR de 40 tonnes côté nord et enrochements en ophite de 10/15 tonnes côté sud), la méthode de levé innovante permet d'apprécier très précisément le profil représentatif du talus et de faire apparaitre les irrégularités de toutes dimensions. Cette technique a permis d'obtenir l'enveloppe numérique précise de la carapace de la digue de Tarnos et des deux digues sud. Assemblées avec le levé bathymétrique multi faisceaux, les mesures permettent d'avoir l'allure générale de l'ouvrage depuis de pied de talus jusqu'au couronnement.

Les résultats obtenus présentent de nombreux avantages par rapport à la technique de levé topographique traditionnelle :

- une meilleure précision du levé de points,

- une densité de points incomparables permettant de choisir a posteriori et à n'importe quels endroits de la digue un nombre illimité de profils à extraire. La 
méthode traditionnelle impose de déterminer un nombre arrêté de profil à lever d'où un surcoût à chaque profil qui n'aurait pas été prévu initialement,

- le faisceau du laser accède aux endroits de l'ouvrage les plus dangereux (pied de talus sur BCR 40 tonnes) alors que le levé traditionnel se limite aux endroits accessibles par l'homme,

- une restitution en 3 dimensions qui est fidèle aux terrains,

- un temps d'exécution réduit à quelques jours pour lever l'ensemble de la digue nord (talus et presse papier) incomparable à une autre méthode,

- un outil de communication très efficace (plan MNT - colorimétrie).

Cette méthode de levé topographique est un outil très puissant et particulièrement intéressant pour établir à un instant $\mathrm{T}$ l'état d'un ouvrage, pour mettre en évidence les désordres présents (recensement exhaustif et quantification très précise) et également pour mettre en place un suivi fin de ce type d'ouvrages.

C'est une technologie en plein développement. Les constructeurs de scanners 3D se livrent actuellement une course au système le plus rapide, le plus précis ou ayant la plus grande portée : certains sont capables d'enregistrer 500000 points à la seconde, d'autres portent à plus de $6000 \mathrm{~m}$. Notons aussi l'émergence très prometteuse de systèmes mobiles, capables d'enregistrer en quelques heures ce qu'un scanner terrestre statique met encore quelques jours à numériser (la précision manque encore au rendez-vous). Cependant, les plus grands progrès restent à faire du coté des logiciels courant du type autocad, afin qu'ils soient rapidement capables de prendre en charge les millions de points d'une campagne de mesure pour offrir aux bureaux d'études toute liberté d'exploitation. Cela ne saurait tarder.

\section{$5 \quad$ Références bibliographiques}

1 CHAZALY B. (2007). La lasergrammétrie appliquée à l'auscultation des ouvrages d'art. Revue XYZ, n 107, pp 18-21.

2 CIRIA, CUR, CETMEF (2007). The Rock Manual. The use of rock in hydraulic engineering ( $2^{\text {nd }}$ edition). CIRIA, Londres, $1200 \mathrm{p}$. 